九州大学学術情報リポジトリ

Kyushu University Institutional Repository

\title{
Aggregation Pheromone Secretion Change of Riptortus pedestris (Hemiptera: Alydidae) Depending on Diet
}

\section{Shin, Hyo Seob}

Department of Applied Biology, College of Agriculture and Life Sciences, Chungnam National University

\section{Ryu, Tae Hee}

Department of Applied Biology, College of Agriculture and Life Sciences, Chungnam National University

\section{Kwon, Hye Ri}

Department of Applied Biology, College of Agriculture and Life Sciences, Chungnam National University

Seo, Mi Ja

Department of Applied Biology, College of Agriculture and Life Sciences, Chungnam National University

他

https://doi.org/10.5109/1526395

出版情報: 九州大学大学院農学研究院紀要. 60 (2)，pp.357-362，2015-09-18. Faculty of Agriculture, Kyushu University

バージョン :

権利関係 : 


\title{
Aggregation Pheromone Secretion Change of Riptortus pedestris (Hemiptera: Alydidae) Depending on Diet
}

\author{
Hyo Seob SHIN ${ }^{1}$, Tae Hee RYU ${ }^{1}$, Hye Ri KWON ${ }^{1}$, Mi Ja SEO ${ }^{1}$, Yong Man YU ${ }^{1}$, \\ Chisa YASUNAGA-AOKI* and Young Nam YOUN ${ }^{1 *}$
}

\author{
Laboratory of Insect Pathology and Microbial Control, Institute of Biological Control, \\ Faculty of Agriculture, Kyushu University, Fukuoka 812-8581, Japan \\ (Received May 7, 2015 and accepted May 19, 2015)
}

\begin{abstract}
To confirm the difference in aggregation pheromone levels in Riptortus pedestris depending on host plants, $R$. pedestris was measured after feeding 6 legumes: mung bean [Vigna radiata var. radiata (L.) R. Wilczek], small black bean (Rhynchosia nulubilis), hairy vetch (Vicia villosa Roth), yellow soybean [Glycine max (L.) Merr.], cranberry bean (Phaseolus vulgaris), and black soybean [Glycine max (L.)]. For the $R$. pedestris collected at bean fields, hairy vetch field, and outdoor field, the pheromones were also analyzed using gas chromatography. We focused on tetradecyl isobutyrate (TI) which is a known insect attractant. The bugs provided with cranberry beans secreted the most TI and those provided with hairy vetch secreted the least TI; the amount of TI secreted was different for each of the beans. The bean bugs' TI secretion percentage was the highest for those that ingested black soybean and lowest for those that ingested hairy vetch. Aggregation pheromone secretions were affected by food sources; with cases of supporting plants displaying the lowest amount of secretion, whereas cases of supporting plants and seeds displayed the highest amount of secretion as well as the highest percentage of secreting bean bugs. Bean bugs captured in bean fields secreted the highest amount of TI component.
\end{abstract}

Key words: Aggregation pheromone, Riptortus pedestris, Secretion change

\section{INTRODUCTION}

Riptortus pedestris causes massive damage to soybeans as well as other crops. Plant damage attributed to $R$. pedestris is found in persimmons and citrus orchards. It has been reported that $R$. pedestris occurs in foxtail millet, proso millet, and sorghum as a dominant species (Chung et al., 1995; Choi et al., 2000; Lee et al., 2009; Kim et al., 2010). As R. pedestris has high mobility and female adults have an excellent ability to distinguish host plants from various crops (Ikeura and Kuroda, 2013), it moves to the neighboring woods or fields when chemical control is applied and returns later. Thus, controlling this pest is difficult. The aggregation pheromone trap has proven to be effective and has been recently used in order to attract and capture $R$. pedestris (Masuta et al., 2001; Huh and Park, 2006; Park et al., 2012; Shin et al., 2012).

Aggregation pheromone of $R$. pedestris secreted by male adults can attract conspecific adults as well as nymphs (Numata et al., 1990; Leal et al., 1995). Aggregation pheromone is composed of (E)-2-hexenyl (Z)-3-hexenoate (E2HZ3H), (E)-2-hexenyl (E)-2hexenoate (E2HE2H) and tetradecyl isobutyrate (TI) (Leal et al., 1995). It has been reported that the composition of aggregation pheromone may vary depending on geographical locations (Leal et al., 1995). Among its components, TI is the main component to which $R$. pedestris is attracted while E2HE3H is a component

\footnotetext{
1 Department of Applied Biology, College of Agriculture and Life Sciences, Chungnam National University, Daejon 305-764, Korea

* Joint corresponding authors (E-mail: youngnam@cnu.ac.kr; yasunaga@grt.kyushu-u.ac.jp)
}

that attracts egg parasitoids such as Ooencyrtus nezarae (Hymenopetra: Encyrtidae) (Leal et al., 1995; Mizutani et al., 1997; Masuta et al., 2001; Huh and Park, 2005). According to a study on the secretion properties of the aggregation pheromone of $R$. pedestris, the secretion increases as days pass and as temperature increases (Huh et al., 2009). Resting and sexually immature males do not secrete E2HZ3H and E2HE2H, and the amount of secretion varies depending on the feeding condition (Mizutani et al., 2008).

In previous studies, it has been reported that the daily growth and survival rate of $R$. pedestris vary depending on soybean cultivars, and that the host preference and degree of damage caused by the bugs also vary depending on traits such as pubescence and pod and hilum color (Son et al., 2000). Many other studies have also reported that secretion of the aggregation pheromone is dependent on the surrounding environment and on physiological conditions in other bean bugs. However, no studies on the differences in aggregation pheromone secretions depending on soybean cultivars have been conducted.

Therefore, in this study, we measured the amount of aggregation pheromone under different feeding conditions in each soybean cultivar to determine how they affected the variation in the amount of aggregation pheromone secreted by $R$. pedestris.

\section{MATERIALS AND METHODS}

\section{Culture and collection of test insect}

Culture of test insect

$R$. pedestris was cultured over successive generations in an acrylic cage $(400 \times 440 \times 500 \mathrm{~mm})$ in the insect 
culture room $\left(25 \pm 1^{\circ} \mathrm{C}, 14 \mathrm{~L}: 10 \mathrm{D}\right)$. Wild type insects were irregularly added in order to prevent inbreeding depression. A layer of paper towel was placed in the acrylic cage to easily remove secretions. The insects were fed soybean cultivars and plants. Seeds from the six legumes used in this experiment were mixed and placed on a Petri dish (150 mm). Cranberry beans (Phaseolus vulgaris) were supplied as foliated plants without pods. In addition, eggs were collected by placing cotton wool in the cage. A water-filled Falcon tube $(50 \mathrm{ml})$ topped with cotton wool was placed in the cage as water source.

All adults used in the experiment were 7-8 days old. Nymphs in unknown life stages were randomly selected and used for the preference experiments.

\section{Collection of test insects}

Field populations were collected by using pheromone traps and a sweeping net in a soybean field $\left(1,207 \mathrm{~m}^{2}\right.$, $36^{\circ} 22^{\prime} 03.8^{\prime \prime} \mathrm{N} 127^{\circ} 21^{\prime} 10.8^{\prime \prime} \mathrm{E}$ ) in Gung-dong and a hairy vetch field $\left(1,070 \mathrm{~m}^{2}, 36^{\circ} 21^{\prime} 40.5^{\prime \prime} \mathrm{N} 127^{\circ} 19^{\prime} 33.8^{\prime \prime} \mathrm{E}\right)$ in Noeun-dong, both in Yuseong-gu, Daejeon. Straightline distance between the collection sites was $2.51 \mathrm{~km}$. Test insects were also caught in various places of the hills near the Chungnam National University in Gung-dong. For the collection using pheromone traps, four pheromone traps were installed at $50 \mathrm{~m}$ intervals between the traps in each test field. Bean bugs were collected from the traps every two weeks. For collection using a sweeping net, it was swept for 10 cycles and repeated three times in each field.

\section{Experimental plants and culture}

Six legumes (Fabaceae) without pods including mung bean, small black bean, hairy vetch, yellow soybean, cranberry bean, and black soybean were used in the laboratory assay. Plants were cultured in a growth chamber $\left(25 \pm 1^{\circ} \mathrm{C}, 14 \mathrm{~L}: 10 \mathrm{D}\right)$. The size and shape of leaves varied depending on the legumes, although legumes were all 2-3 weeks old from the seeding.

Aggregation pheromone extraction and GC analysis Aggregation pheromone extraction and analysis

Aggregation pheromone of $R$. pedestris was extracted from one male adult as follows. First, it was placed and soaked in $1 \mathrm{~mL}$ Hexane (99\%, Merck, Germany) in $1.5 \mathrm{ml}$ micro tube for 5 minutes. Gas chromatography (7890A GC Chemstation, Agilent Technologies, USA) was used for GC analysis. Conditions described in the study of Huh et al. (2008) were modified for the analysis to create quantitative curves and measure the amount of secretion. DB-WAX column (ID $0.25 \mathrm{~mm} \times 30 \mathrm{~m}$, Agilent Technologies, USA) was used in this experiment. GC conditions are as follows. We increased the oven temperature up to $210^{\circ} \mathrm{C}$ by $10^{\circ} \mathrm{C} /$ minute after it was maintained at $50^{\circ} \mathrm{C}$ for 1 minute. Then, it was maintained for $15 \mathrm{~min}-$ utes. Carrier gas was $\mathrm{H}_{2}$ and the inlet temperature was $250^{\circ} \mathrm{C}$. A detector was FID and temperature was $250^{\circ} \mathrm{C}$.

Creation of quantitative curves of each component

In this study, standards were ordered from Practica
Chem Company and used to analyze the three main pheromone components, E2HZ3H, E2HE2H and TI, among other components of aggregation pheromone. Purity was 95\%, 92\% and 95\%, respectively. GC conditions were used for the quantitative analysis on the same three components. On the basis of results, quantitative curves were created for each component. After the aggregation pheromone lure was diluted with Hexane (99\%, Merck, Germany) solution to make 1, 0.5, 0.1, 0.05, 0.01, 0.005, and $0.001 \mathrm{mg} / \mathrm{ml}, \mathrm{DB}-W A X$ column was applied. Curves were created by using the area values and concentrations on the GC graph; these curves were then used to calculate aggregation pheromone secretions. Every curve's determination coefficient value was 0.99 and above.

\section{Difference in amount of aggregation pheromone secretion in each legume}

We determined the difference in the amounts of aggregation pheromone secretion of $R$. pedestris fed with six legumes. Six podless legume plants were placed in each acrylic cage $(400 \times 400 \times 500 \mathrm{~mm})$ and 10 male adults of $R$. pedestris were put in a cage and cultured for 10 days. Then, aggregation pheromone was extracted and quantified. Mating status and body weight were unknown for the tested bugs. Extraction methods and conditions for GC analysis are as mentioned above. Experiments were repeated a total of five times.

\section{Difference in the amount of secretion based on feeding conditions}

Cranberry bean seeds, foliated plants without pods, and plants with seeds were placed in each rearing cage to examine how the difference in the amount of pheromone secretion depended on feeding conditions. Each of the conditions used one plant and 100 seeds. The waterfilled tube was placed in the acrylic cage as described above. Ten male adults of $R$. pedestris were placed in the cage and were cultured for seven days before the extraction of aggregation pheromone. Mating status and body weight are unknown for the tested bugs. The extraction method and conditions for GC analysis are described above. Experiments were repeated five times.

\section{Comparison of difference in aggregation pherom- one secretion aggregation pheromone secretion in each crop of bean bugs caught in the bean field, hairy vetch field and outdoor field}

Test insects were obtained by sweeping an insect net in the bean field, hairy vetch field and outdoor field. As various soybean cultivars were mixed in the bean field, bean bugs were captured during the period when pods were made and harvested after planting. In the case of hairy vetch, it was planted in the middle of April and pods were made in the middle of June. Bean bugs were captured during the period when hairy vetch seeds feel and regenerated. In the outdoor bean field, bean bugs were captured in the area which was farthest from the bean field or hairy vetch field and where other kinds of crops (pepper, Proso Millet, etc.) grew. 


\section{Statistical analysis}

Statistical analysis used in this experiment is as follows: When the distribution of aggregation pheromone secretion depending on soybean cultivars and variation in amount of aggregation pheromone secretion depending on the number of bean bugs were analyzed, graphs were created using a Sigma plot 12.0. When the aggregation pheromone secretion resulting from the feeding conditions and soybean cultivars and the secretions from each crop in the outdoor field were analyzed, a one-way ANOVA test was performed by using an SPSS (PASW statistics 20.0) within the range of significant probability of 0.05 . A significance test was performed through Tukey's HSD test.

\section{RESULTS}

\section{Difference in aggregation pheromone secretion based on legumes}

The bug that ingested the black soybeans secreted highest amount of E2HZ3H while hairy vetch had the smallest amount of secretion of E2HZ3H. Mung bean had the largest amount of E2HE2H secretion and small black bean had the smallest amount of E2HE2H secretion. In addition, TI has been known to be the most associated with the attractiveness of aggregation pheromone to $R$. pedestris. Cranberry bean had the largest amount of TI secretion, followed in decreasing order by small black bean, mung bean, black soybean, yellow soybean, and hairy vetch (Table 1 ).

According to the following results, the secretion of three main components of aggregation pheromone except E2HE2H was statistically significant in six legumes. For the ratio of bean bugs secreting each component, more than $90 \%$ of bean bugs used in the experiment secreted E2HZ3H and E2HE2H in all tests. However, bean bugs that secreted TI showed a difference of percentage for each legume. Bean bugs that secreted TI showed the highest percentage for black soybean and showed the lowest percentage for hairy vetch (Fig. 1).

When looking at the composition ratio (E2HZ3H: E2HE2H:TI) in aggregation pheromone secreted from bean bugs used in the experiment, cranberry bean showed the highest ratio for TI in 1:1.51:0.54 and hairy vetch showed the lowest ratio in 1:1.77:0.12 (Table 2).

According to the results of experiment, there were variations in the amount of aggregation pheromone produced by $R$. pedestris between the six legumes used in the experiment.

Table 1. Difference of aggregation pheromone components ( $\mu \mathrm{g} / \mathrm{bug} \pm \mathrm{SE})$ of $R$. pedestris according to host seeds and foliated plants

\begin{tabular}{llll}
\hline \multicolumn{1}{c}{ Plant } & \multicolumn{1}{c}{ E2HZ3H* } & E2HE2H & TI \\
\hline Cranberry bean & $12.01 \pm 5.45 \mathrm{ab} * *$ & $18.15 \pm 8.71 \mathrm{a}$ & $6.55 \pm 3.04 \mathrm{a}$ \\
Small black bean & $10.69 \pm 3.41 \mathrm{ab}$ & $17.30 \pm 4.93 \mathrm{a}$ & $3.16 \pm 0.92 \mathrm{bc}$ \\
Mung bean & $13.23 \pm 3.45 \mathrm{ab}$ & $22.69 \pm 6.37 \mathrm{a}$ & $5.70 \pm 2.41 \mathrm{abc}$ \\
Yellow soybean & $13.98 \pm 4.64 \mathrm{a}$ & $22.12 \pm 7.46 \mathrm{a}$ & $4.36 \pm 2.69 \mathrm{ab}$ \\
Black soybean & $14.17 \pm 2.72 \mathrm{a}$ & $22.56 \pm 4.18 \mathrm{a}$ & $4.32 \pm 2.31 \mathrm{ab}$ \\
Hairy vetch & $10.43 \pm 4.99 \mathrm{~b}$ & $18.48 \pm 7.56 \mathrm{a}$ & $1.26 \pm 0.21 \mathrm{c}$ \\
\hline
\end{tabular}

*E2HZ3H: (E)-2-hexenyl (Z)-3-hexenoate, E2HE2H: (E)-2-hexenyl (E)-2-hexenoate, TI: tetradecyl isobutyrate. **Test by Tukey's HSD $(\alpha=0.05)$ in SPSS (PASW statistics 20.0).

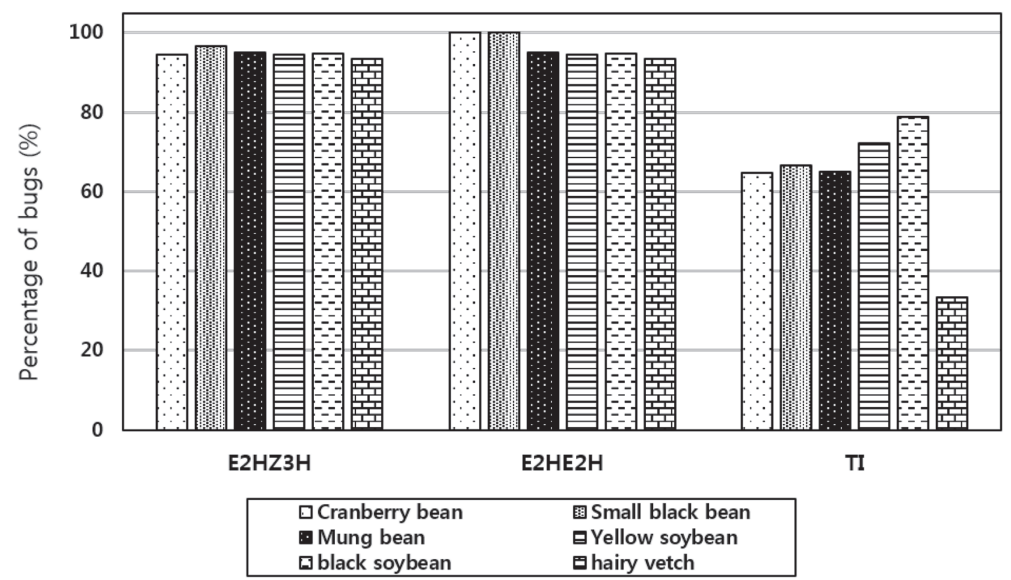

Fig. 1. Percentage of $R$. pedestris that secreted the three major components of aggregation pheromone according to the host plants. 
Table 2. Aggregation pheromone components ratio of $R$. pedestris according to host plant and different field

\begin{tabular}{ccc}
\hline & $\begin{array}{r}\text { Ratio of aggregation pheromone components } \\
\left(\mathrm{E} 2 H Z 3 H^{*}: \text { E2HE2H }: \text { TI) }\right.\end{array}$ \\
\hline Cranberry bean & $1: 1.51: 0.54$ \\
Slant & Mung bean & $1: 1.62: 0.29$ \\
& Yellow soybean & $1: 1.72: 0.43$ \\
& Black soybean & $1: 1.58: 0.31$ \\
& Hairy vetch & $1: 1.59: 0.30$ \\
Field & Hairy vetch & $1: 1.77: 0.12$ \\
\hline & Bean & $1: 1.53: 0.12$ \\
& Other & $1: 1.84: 0.32$ \\
\hline
\end{tabular}

*E2HZ3H: (E)2-hexenyl (Z)-3-hexenoate, E2HE2H: (E)-2-hexenyl (E)-2-hexenoate, TI: tetradecyl isobutyrate.

Table 3. Amount of aggregation pheromone secreted ( $\mu \mathrm{g} / \mathrm{bug} \pm \mathrm{SE}$ ) by $R$. pedestris according to different feed sources

\begin{tabular}{cccc}
\hline & E2HZ3H* & E2HE2H & TI \\
\hline Plant & $7.19 \pm 3.43 \mathrm{~b}^{* *}$ & $13.74 \pm 7.48 \mathrm{a}$ & $0.49 \pm 0.05 \mathrm{a}$ \\
Seed & $12.88 \pm 3.55 \mathrm{a}$ & $18.99 \pm 5.99 \mathrm{a}$ & $3.85 \pm 2.03 \mathrm{ab}$ \\
Plant + Seed & $12.01 \pm 5.45 \mathrm{a}$ & $18.14 \pm 8.70 \mathrm{a}$ & $6.55 \pm 3.04 \mathrm{~b}$ \\
\hline
\end{tabular}

*E2HZ3H: (E)-2-hexenyl (Z)-3-hexenoate, E2HE2H: (E)-2-hexenyl (E)-2-hexenoate, TI: tetradecyl isobutyrate. **Test by Tukey's HSD $(\alpha=0.05)$ in SPSS (PASW statistics 20.0).

\section{Difference in aggregation pheromone secretion based on feeding conditions}

When plants, seeds and plants mixed with seeds were supplied, the amount of aggregation pheromone secreted by test bean bugs was compared for each group (Table 3).

It was found that E2HZ3H, E2HE2H and TI showed the lowest amount of secretion when the bugs only ingested plants. The ratio of bean bugs that secreted the pheromone was also the lowest. When seeds were supplied in the diet, production of E2HZ3H and E2HE2H and the ratio of bugs that secreted the pheromone were similar. However, TI showed the most statistically significant difference for each feeding condition. Therefore, the above results suggest that there are some variations in the amount of aggregation pheromone secreted from $R$. pedestris that are depending on diets.

\section{Comparison of difference in aggregation pherom- one secretion in each crop of bean bugs caught in the field}

The amount of aggregation pheromone secretion from each bean bug captured in the field with various crops by using insect nets was compared. Both E2HZ3H and E2HE2H were detected in bean bugs captured in the outdoor field, but TI was not detected. Nevertheless, all three components were detected in bean bugs captured in the hairy vetch and bean fields. The secretions from bean bugs captured in the bean field were the same as those from bean bugs captured in the hairy vetch field

Table 4. Amount of aggregation pheromone secreted ( $\mu \mathrm{g} / \mathrm{bug} \pm \mathrm{SE}$ ) by $R$. pedestris caught in different collection fields

\begin{tabular}{cccc}
\hline Field & E2HZ3H* & E2HE2H & TI \\
\hline Hairy vetch & $8.91 \pm 3.63 \mathrm{a}^{* *}$ & $13.60 \pm 7.33 \mathrm{ab}$ & $1.09 \pm 0.30 \mathrm{a}$ \\
Bean & $9.88 \pm 4.23 \mathrm{a}$ & $18.22 \pm 8.95 \mathrm{a}$ & $3.18 \pm 1.95 \mathrm{a}$ \\
Other & $7.44 \pm 3.86 \mathrm{a}$ & $11.05 \pm 7.11 \mathrm{~b}$ & - \\
\hline
\end{tabular}

*E2HZ3H: (E)-2-hexenyl (Z)-3-hexenoate, E2HE2H: (E)-2-hexenyl (E)-2-hexenoate, TI: tetradecyl isobutyrate. **Test by Tukey's HSD $(\alpha=0.05)$ in SPSS (PASW statistics 20.0) 
(Table 4).

When looking at the ratio of the three components of aggregation pheromone, bean bugs captured in the hairy vetch field and bean field secreted the ratio of 1:1.53:0.12 and 1:1.84:0.32, respectively (Table 2). Taken all results together, it was found that bean bugs captured in the bean field secreted different amounts of aggregation pheromone than those captured in the outdoor bean field or hairy vetch field did.

\section{DISCUSSION}

In this study, we investigated the variation in the amount of aggregation pheromone secretion aggregation pheromone secretion from a bean bug, $R$. pedestris (Hemiptera: Alydidae), depending on diets by using GC-MS.

Aggregation pheromone of $R$. pedestris consists of E2HZ3H, E2HE2H and TI. Since then, there have been recent reports that Octadecyl isobutyrate (OI) is included. Research shows that OI, combined with the other three components, plays a role to increase the attractiveness (Yasuda et al., 2007a). However, it was not detected for any of the six legumes in this experiment and there was no similarity between bean bugs secreting aggregation pheromone. In addition, it has been found that (E)-2hexanyl hexanoate, known as the alarm pheromone, creates a synergistic effect along with TI and thus facilitates the secretion of aggregation pheromone (Yasuda et al., 2007b).

When quantitative analysis was performed on aggregation pheromone, we focused on TI, a known insect attractant. E2HZ3H and E2HE2H were measured at a certain amount or higher and the ratio of bean bugs secreting them was high in all experiments. However, there were variations in the secretion of TI among the six legumes. TI secretion also varied depending on diets. The ratio of aggregation pheromone components varied depending on different geographical locations. When the ratio of the three components was normalized by the amount of E2HZ3H $(=1)$, the ratio of Tsukuba and Kumamoto populations in Japan was 1:2.8:0.2 and 1:1.5:0.1, respectively. In addition, the ratio of Jinju and Iksan populations in Korea was 1:1.4:0.2 and 1:0.8:0.2, respectively (Leal et al., 1995; Huh et al., 2008). The results of analysis on the bean bugs captured in the outdoor bean field in Daejeon indicated that the composition ratio was 1:1.84:0.32, which was different from that in Japan but similar to that of the Jinju population (Table 2).

As in other bugs, the amount of aggregation pheromone secretion from $R$. pedestris varied depending on the surrounding environment, the bugs' physiological state, or their nutritional state (Mizutani et al., 2008). Male adults do not secrete aggregation pheromone when there are no foods available, but they secrete it to attract other populations when foods are available (Leal et al., 1995). Male adults attracted to live bait males did not have foods in their stomachs and TI was not detected (Mizutani et al., 2008). For this reason, bean bugs captured by using pheromone traps or sweeping showed small amounts of aggregation pheromone regardless of their field of origin. This suggested that bean bugs that were attracted to pheromone traps seemed to eat less food. According to the studies of Huh and Park (2006) and Shin et al. (2012), when additional foods were supplied in a pheromone trap, the trap's attractiveness increased. Persimmon and soybeans showed greater damage when they were supplied together rather than when only persimmon was supplied (Lee et al., 2009). The previous study indicated that when $R$. pedestris was cultured under the single condition (10L:14D), male adults that entered reproductive dormancy secreted aggregation pheromone. However, the function of aggregation pheromone secretion was not directly associated with mating (Wada et al., 1997). The amount of aggregation pheromone secretion was not different, regardless of mating, weight, or time zone. However, it varied depending on the number of elapsed days and the time of capture. (Huh et al., 2009).

Both mung bean and hairy vetch were legumes which are known to receive damage from $R$. pedestris. However, when those two legumes were supplied as foods, nymph could not complete their metamorphosis into adults, resulting in an incomplete life cycle. Therefore, these legumes were not suitable for foods (Bae et al., 2009; Seo et al., 2011). Despite the small amount of aggregation pheromone in hairy vetch fields, $R$. pedestris could infest and inflict damage on hairy vetch. They may use the hairy vetch field for a temporary food source due to the lack of other available food items. However, mung bean increased the aggregation pheromone secretion even though it contained resistant materials affecting the growth of $R$. pedestris (Jung et al., 2005). In the future, we would like to examine the components of this study's test legumes and investigate the correlation between these components and the synthesis of aggregation pheromone in $R$. pedestris.

\section{ACKNOWLEDGEMENTS}

This work was carried out with the support of "Cooperative Research Program for Chungnam National University (Project No. is 2014-1194-01)”.

\section{REFERENCES}

Bae, S. D., H. J. Kim, Y. N. Yoon and B. R. Choi 2009 Effects of a mung bean cultivar, Jangannogdu on nymphal development, adult longevity and oviposition of soybean stink bugs. Kor. J. Appl. Entomol., 48: 311-318

Choi, D. S., K. C. Kim and K. C. Lim 2000 The status of spot damage and fruit piercing pests on Yuzu (Citrus junos) fruit. Kor. J. Appl. Entomol., 39: 259-266

Chung, B. K., S. W. Kang and J. H. Kwon 1995 Damages, occurrences and control of hemipterous insects in non-astringent persimmon orchards. RDA J. Agri. Sci., 37: 376-382

Huh, H. S., S. A. Jang and C. G. Park 2009 Variation in aggregation pheromone secretion of bean bug, $R$. clavatus. Kor. J. Appl. Entomol., 48: 73-79

Huh, H. S., J. E., Yun, T., Wada, N. Mizutani and C. G. Park 2008 Composition of the aggregation pheromone components of 
Korean bean bug and attractiveness of different blends. Kor. J. Appl. Entomol., 47: 141-147

Huh, W. and C. G. Park 2005 Seasonal occurrence and attraction of egg parasitoid of bugs, Ooencyrtus nezarae, to aggregation pheromone of bean bug, R. clavatus. Kor. J. Appl. Entomol., 44: $131-137$

Huh, W. and C. G. Park 2006 Increased attractiveness of the aggregation pheromone trap of bean bug, $R$. clavatus. Kor. J. Appl. Entomol. , 45: 87-90

Ikeura, H. and K. Kuroda 2013 Preference by adult female $R$. Clavatus Thunberg for plants from the fabaceae and plant families. J. Agri. Sustainability., 3: 205-215

Jung, J. K., J. K. Moon and B. Y. Seo 2005 Screening of leguminous germplasm for resistance against the bean bug, $R$. clavatus. Treat. Crop Res., 7: 639-666

Kim, J. S., H. C. Ko, S. T. Yoon, Y. H. Cho and J. G. Kim 2010 Occurrence of insect pest from organic seed producing field of minor grain germplasms. Kor. J. Crop Sci., 55: 58-64

Leal, W. S., H. Higuchi, N. Mizutani, H. Nakamori, T. Kadosawa and M. Ono 1995 Multifunctional communication in $R$. clavatus (Heteroptera: Alydidae): Conspecific nymphs and egg parasitoid Ooencyrtus nezarae use the same adult attractant pheromone as chemical cue. J. Chem. Ecol., 21: 973-985

Lee, H. S., B. K. Chung, T. S. Kim, J. H. Kown, W. D. Song and C. W. Rho 2009 Damage of sweet persimmon fruit by the inoculation date and number of stink bugs, $R$. clavatus, Halyomorpha halys and Plautia stali. Kor. J. Appl. Entomol., 48: 485-491

Masuta, S., N. Mizutani and T. Wada 2001 Difference in response of $R$. clavatus (Thunberg) (Heteroptera: Alydidae) and its egg parasitoid Ooencyrtus nezarae Ishii (Hymenoptera: Encyrtidae) to the synthetic aggregation pheromone of $R$. clavatus. Jap. J. Appl. Entomol. Zool., 45: 215-218

Mizutani, N., T. Wada, H. Higuchi, M. Ono and W. S. Leal 1997 A component of a synthetic aggregation pheromone of $R$. clavatus (Thunberg) (Heteroptera: Alydidae), that attracts an egg parasitoid, Ooencyrtus nezarae Ishii (Hymenoptera: Encyrtidae). Appl. Entomol. Zool., 32: 504-507

Mizutani, N., T. Yasuda, T. Yamaguchi and S. Moriya 2008
Pheromone contents and physiological conditions of adult bean bugs, Riptortus pedestris (Heteroptera: Alydidae), attracted to conspecific males during non-diapause and diapause periods in fields. Appl. Entomol. Zool., 43: 331-339

Numata, H., M. Kon and T. Hidaka 1990 Male adults attract conspecific adults in the bean bug, $R$. clavatus Thunberg (Heteroptera: Alydidae). Appl. Entomol. Zool., 25: 144-145

Park, C. G., K. H. Yum and J. K. Jung 2012 Damage reduction effect and attracted distance by aggregation pheromone trap of the bean bug, Riptortus pedestris (Fabricius), (Hemiptera: Alydidae) in soybean fields. Kor. J. Appl. Entomol., 51: 411419

Seo, M. J., H. R. Kwon, K. S. Yoon, M. A. Kang, M. W. Park, S. H. Jo, H. S. Shin, S. H. Kim, E. J. Kang, Y. M. Yu and Y. N. Youn 2011 Seasonal occurrence, development and preference of Riptortus pedestris on hairy vetch. Kor. J. Appl. Entomol., 50: $47-53$

Shin, Y. H., S. H. Yun, Y. U. Park, J. J. An, C. M. Yoon, Y. N. Youn and G. H. Kim 2012 Seasonal fluctuation of Riptortus pedestris (Hemiptera: Alydidae) in Chungbuk Province. Kor. J. Appl. Entomol., 51: 99-109

Son, C. K., S. G. Park, Y. H. Hwang and B. S. Choi 2000 Field occurrence of stink bug and its damage in soybean. Kor. J. Crop Sci., 45: 405-410

Wada, T., N. Mizutani and H. Higuchi 1997 Aggregation pheromone of the bean bug, $R$. clavatus Thunberg (Heteroptera: Coreidae): Mating behavior and pheromone release in male adults. Proc. Asso. Plant Prot. Kyushu, 43: 82-85

Yasuda, T., N. Mizutani, N. Endo, T. Fukuda, T. Matsuyama, K. Ito, S. Moriya and R. Sasaki 2007a A new component of attractive aggregation pheromone in the bean bug, $R$. clavatus (Thunberg) (Heteroptera: Alydidae). Appl. Entomol. Zool. 42: $1-7$

Yasuda, T., N. Mizutani, Y. Honda, N. Endo, T. Yamaguchi, S. Moriya, T. Fukuda, and R. Sasaki 2007b A supplemental component of aggregation attractant pheromone in the bean bug $R$. clavatus (Thunberg) (Heteroptera: Alydidae), related to food exploitation. Appl. Entomol. Zool., 42: 161-166 